\title{
Modelo Conceitual para Sistemas de Recomendação voltados a Governo Eletrônico com o uso do Cadastro Base do Cidadão
}

\author{
Aline M. Moisinho' ${ }^{1}$ Emilly G. M. da Silva ${ }^{1}$, Paulo F. Silva Jr¹, \\ Daniel Schneider ${ }^{1}$, Claudia L. R. Motta ${ }^{1}$
}

1Programa de Pós-Graduação em Informática - Universidade Federal do Rio de Janeiro (UFRJ) - Rio de Janeiro - RJ - Brasil

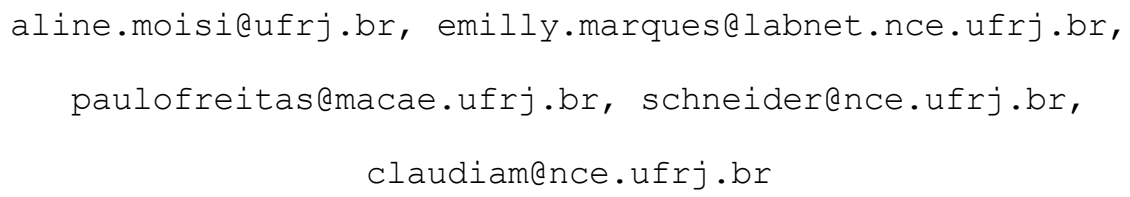

\begin{abstract}
This paper proposes a conceptual model for the development of recommendation systems aimed at e-government services to citizens, using personalization techniques and the "Cadastro Base do Cidadão", in addition to the notion of their self-profile. Ethical issues related to the processes of collecting and processing large amounts of data are discussed and, based on that, the self-profile is proposed, aiming to guarantee the user the right of informational self-determination. The model follows a concept of a hybrid recommendation system with reactive and proactive solutions.
\end{abstract}

Resumo. Este artigo propõe um modelo conceitual para o desenvolvimento de sistemas de recomendação voltado a serviços de governo eletrônico aos cidadãos, utilizando técnicas de personalização e o "Cadastro Base do Cidadão", junto com a noção de auto perfil. Questões éticas relacionadas aos processos de coleta e tratamento de grandes quantidades de dados são discutidas e, a partir disso, é proposto o auto perfil, visando garantir ao usuário o direito de autodeterminação informacional. $O$ modelo segue um conceito de sistema de recomendação híbrido e com soluções reativas $e$ proativas.

\section{Introdução}

O governo de diversos países têm criado portais governamentais com o objetivo de melhorar os serviços prestados aos cidadãos, ampliando o canal de comunicação, trazendo transparência e contribuindo para a redução de custos e controle de fraude. No Brasil, mais de $60 \%$ dos serviços prestados aos cidadãos podem ser realizados de forma totalmente digital, de acordo com dados encontrados no portal do governo [Governo do Brasil 2021], [Governo de Quebec 2021].

Em paralelo e de forma consonante à estratégia de ampliar a oferta de serviços de governo digital, o Poder Executivo Federal brasileiro está trabalhando na construção do Cadastro Base do Cidadão (CBC). Trata-se de uma base de dados construída a partir da integração de dados provenientes de outras bases estatais, contando com informações adquiridas por distintos órgãos do governo federal e reunindo informações como: CPF, nome, data de nascimento, sexo, filiação, nacionalidade, naturalidade e informações de vários entes da federação, secretarias, ministérios, entre outros [Governo do Brasil/a 2021].

Embora a progressiva capacidade técnica de coleta e tratamento de dados possa trazer muitos benefícios aos cidadãos em termos de prestação de serviços, desenvolvimento social e 
econômico; entidades internacionais como a ONU têm demonstrado grande preocupação no que diz respeito à proteção dos direitos dos consumidores e dos cidadãos em relação aos seus dados pessoais [Ferreira 2020]. Essas advertências devem ser objeto de reflexão pois nos colocam diante de questionamentos éticos, jurídicos e técnicos a respeito da propriedade e utilização de dados pessoais, sendo esse um dos focos do presente trabalho.

Ademais, com vistas à satisfação dos usuários em relação a prestação de serviços disponíveis no portal do governo eletrônico, é proposto um modelo conceitual para o desenvolvimento de sistemas de recomendação voltados a serviços de governo eletrônico aos cidadãos. O objetivo é que o sistema possa recomendar serviços personalizados a partir da integração do auto perfil com as informações presentes no CBC.

O sistema de recomendação é uma eficiente aplicação para fornecer serviços de informação mais inteligentes e proativos aos seus usuários. Eles combinam várias técnicas e algoritmos computacionais de aprendizado de máquina com informações extraídas dos perfis do usuário e selecionam itens personalizados com base no estudo do interesse dos usuários de acordo com o contexto em que estão inseridos, dessa forma ele é considerado a aplicação de personalização mais popular que existe. Eles recomendam itens (música, livros, artigos, viagens, etc) ou serviços que possam ser relevantes para o usuário de acordo com sua necessidade [Cazella et al. 2010].

Dessa forma, utilizar um sistema de recomendação que integre a noção de auto perfil ao Cadastro Base do Cidadão (CBC) para serviços de governo eletrônico pode ser uma solução para oferecer serviços personalizados aos cidadãos, garantindo proteção à privacidade de dados e livre expressão da identidade pessoal em meio virtual. O objetivo é que o sistema de recomendação descarte os serviços irrelevantes dos resultados de busca, apresentando apenas resultados relevantes de interesse do usuário e de acordo com a construção do seu auto perfil no portal do governo.

Com base nos objetivos gerais, o presente estudo pode ser situado na categoria de pesquisa exploratória, pois visa proporcionar uma maior aproximação ao problema, bem como o aprimoramento de ideias [Gil 2002]. Os procedimentos metodológicos adotados foram preponderantemente a pesquisa bibliográfica e a análise exploratória do portal eletrônico do governo do Brasil, com a utilização do Design Science Research Methodology (DSRM) para o desenvolvimento do modelo conceitual. A DSRM se constitui em um processo de projetar artefatos para resolver problemas, avaliar o que foi projetado e comunicar os resultados obtidos. O artefato apresentado neste trabalho se trata de um modelo, ou seja, conjunto de proposições que expressam as relações entre os diversos conceitos de um domínio, e a avaliação chamada de "descritiva" por "argumento informado" foi empregada para sustentar a utilidade do artefato criado [Junior et al. 2017].

Isto posto, o artigo busca debater questões éticas relacionadas ao uso de grandes bases de dados, como o Cadastro Base do Cidadão, por governos e empresas, com o intuito de apresentar soluções capazes de contornar as possíveis problemáticas decorrentes da coleta e tratamento de grande quantidade de dados pessoais. Em seguida é proposto um modelo conceitual para o desenvolvimento de sistemas de recomendação voltados a serviços de governo eletrônico aos cidadãos, utilizando técnicas de personalização e o Cadastro Base do Cidadão, junto com a noção de auto perfil, para recomendar serviços específicos que atendam necessidades do usuário, estimule o acesso aos serviços eletrônicos governamentais e garanta a autodeterminação informativa. 


\section{Trabalhos Relacionados}

Nessa seção iremos discutir alguns trabalhos relacionados ao tema de sistemas de recomendação voltados ao governo eletrônico.

O trabalho de [Ayachi et al. 2016], relata o sistema de recomendação proativo e reativo utilizado no governo de Quebec, que oferece serviços do governo eletrônico personalizado e inteligente por meio de seu portal. Personalizar o perfil do usuário permite que os portais governamentais se tornem mais eficientes, atraindo os cidadãos a utilizarem os serviços online. Outra vantagem da personalização relatada é realizar as recomendações de acordo com as preferências, necessidade e informações geográficas dos cidadãos, ou seja, eles terão acesso às informações relevantes na interface personalizada e se tornarão leais ao portal por oferecer-lhes serviços cada vez mais satisfatórios.

Já o trabalho de [Macedo et al. 2020] apresenta uma discussão sobre a dificuldade de interpretabilidade de um conjunto de dados abertos, realiza a revisão da literatura sobre esses dados e propõe uma ferramenta web gratuita de recomendação de visualização de dados governamentais abertos fornecendo facilidade na criação de visualizações e na melhoria da interpretabilidade e contribuição para a democratização da análise de dados, promove a transparência pública permitindo maior participação do cidadão na manipulação dos dados, porque usuários inexperientes no assunto podem, com poucos cliques, receber uma recomendação de visualização de correlações de padrões e tendências, proporcionando insights relevantes.

Por outro lado, o estudo de [Guo and Lu 2007] propõe uma abordagem para lidar com questões de recomendação de itens únicos em serviços de governo eletrônico, aplicado para melhorar a qualidade dos serviços online de governo para empresas, abordando particularmente questões de recomendação personalizada de feiras de negócios internacionais. Através da integração de técnicas de similaridade semântica e filtragem colaborativa baseada em itens, o sistema de recomendação desenvolvido auxilia as empresas a encontrar feiras de negócio certas para elas, diminuindo custos e riscos envolvidos na seleção, entrada e desenvolvimento de mercados internacionais.

Os trabalhos apresentados contribuem para as discussões a respeito do tema de sistemas de recomendação voltados aos sistemas de governo eletrônico, no que diz respeito à personalização, visualização de dados, perfil de usuário, entre outras questões. Nosso trabalho, por sua vez, apresenta uma proposta focada no cidadão e na autodeterminação informacional, questão não considerada na literatura encontrada.

\section{Serviços no portal do governo eletrônico: perfilização utilizando o Cadastro Base do Cidadão (CBC) e possíveis implicações éticas}

\subsection{Portal do Governo Eletrônico}

Analisando o portal do Governo do Brasil [Governo do Brasil 2021], podemos ver a disponibilização de 4137 serviços de 193 órgãos do governo, onde um pouco mais de 200 serviços são de fato utilizados com $70 \%$ de avaliação positiva e verificamos que $65 \%$ dos serviços totais já são digitais e o tempo médio de espera para retorno do serviço é de 39 dias, além disso, existe uma grande quantidade de informações úteis disponíveis ao cidadão. 
O portal permite realizar consultas informativas a partir de informações que estão disponíveis no próprio portal, onde o usuário não precisa se logar para acessar o que deseja e também fornece a opção de realizar um cadastro para realizar solicitações e acompanhamentos de serviços. Alguns serviços ainda não estão integrados e digitalizados e nesse caso apenas a informação é disponibilizada, mesmo estando na área logada, isso porque o portal não está finalizado, a estratégia do governo digital estima que até o final de 2022 todos os serviços públicos estejam digitalizados [Governo do Brasil/b 2021].

\subsection{Cadastro Base do Cidadão (CBC): implicações éticas no tratamento de dados pessoais em sistemas de recomendação de serviços governamentais}

No âmbito da administração pública federal do Brasil, a governança no compartilhamento de dados é regulamentada pelo Decreto $\mathrm{n}^{\circ}$ 10.046, de 9 de Outubro de 2019, que instituiu também o denominado Cadastro Base do Cidadão (CBC), estabelecendo diretrizes para compartilhamento de dados entre órgãos e entidades da administração pública federal. De acordo com o documento, a formação do CBC tem por finalidade, entre outras coisas, viabilizar a criação de um meio unificado de identificação do cidadão para a prestação de serviços públicos [Brasil/a 2019].

O decreto disciplina a centralização de 51 bases de cadastro nacionais. Autoridades governamentais informam que as medidas previstas no decreto irão otimizar o relacionamento dos cidadãos com as instituições públicas, evitando o preenchimento de novos cadastros para lidar com cada instituição. No entanto, a promulgação do decreto tem gerado suspeitas e temores por parte da opinião pública. Alguns especialistas consideram que pode estar sendo criada uma ferramenta de vigilância estatal, onde o cidadão se tornará refém da obscuridade pela qual a burocracia estatal poderá interligar uma variedade de dados pessoais em nome de uma maior eficiência para prestação de serviços não esclarecida previamente [Ferreira 2020].

A respeito do $\mathrm{CBC}$, certamente ainda é cedo para conclusões nesse sentido, no entanto, tais advertências não devem ser ignoradas e merecem ser objeto de reflexão no que tange a segurança e a proteção dos dados pessoais, bem como para reflexões a respeito da construção de um ambiente virtual adequado ao desenvolvimento da identidade pessoal. Os dados pessoais podem ser entendidos como elemento constituinte da identidade da pessoa e como parte fundamental da personalidade humana [Ferreira 2020], assim, a compreensão de alguns aspectos das teorias acerca da identidade pessoal é útil ao debate ético aqui proposto.

Um importante aspecto de tais teorias diz respeito à abordagem em que a identidade é formada por uma espécie de narrativa, especialmente em primeira pessoa, unindo experiências vividas pelo indivíduo em um todo coerente. Nesse contexto, a privacidade seria indispensável para a conformação da identidade, uma vez que a identidade pessoal seria o resultado interminável da seleção de informações entre o que queremos revelar e o que desejamos guardar em segredo [Martins et al. 2019].

Dessa forma, o controle de dados pessoais, deve envolver o exercício tanto da dimensão negativa dessa narrativa, ao impedir a circulação de determinada informação, quanto de sua dimensão ativa, ao permitir a expressão de informações como sendo sua. Contudo, em ambientes virtuais a dimensão ativa de ter algo a dizer a respeito de sua própria identidade pessoal não recebe a mesma atenção que sua contraparte negativa de garantir a não exposição de informações pessoais [Martins et al. 2019]. Nesse contexto, o desenvolvimento de técnicas de perfilização e tomada de decisão algorítmicas com base em dados pessoais não 
informados podem reproduzir situações danosas ao desenvolvimento da identidade pessoal, pois impedem a dimensão ativa de narrar em primeira pessoa informações sobre si.

Diante disso, é de suma importância pensar de que forma o acúmulo de informações sobre o cidadão, somado à geração de informações por meio das tecnologias de perfilização é capaz de gerar problemas ao exercício da autonomia informacional, com o intuito de refletir sobre possíveis maneiras de contornar essas problemáticas. Na medida em que o processo de construção do perfil de usuário visa obter conhecimento sobre as preferências individuais que determinam o comportamento, coletando dados a partir de informações implícitas e explícitas [Montaner et al. 2003], muitas vezes não há espaço para a construção autônoma da própria identidade em ambientes virtuais, pois através de técnicas de mineração de dados preferências e comportamento podem ser predizíveis por parte de sistemas algorítmicos. Ou seja, o usuário tem pouco (ou nenhum) controle sobre como é "visto" [Martins 2019].

Tendo em vista que sistemas de recomendação são aplicações que fazem uso de técnicas de perfilização e mineração de dados para personalização de serviços, em alguns casos esse processo acaba por criar relatos sobre os indivíduos sem interações diretas entre os usuários e o sistema, ou seja, muitas vezes o perfil do usuário é criado sem a participação do mesmo em trazer informações sobre si. Ademais, essas técnicas não atuam em escala individual, considerando apenas os dados obtidos de um único indivíduo, muitas vezes os perfis são construídos a partir da fusão de dados de vários usuários, o que possibilita inferir informações sobre o indivíduo que ele não quer revelar, tendo como fundo as noções de comportamento coletivo ou de grupo para tais inferências [Martins 2019].

Isto posto, acreditamos que a dimensão ativa para a construção de identidades virtuais deve ser levada em consideração no processo de desenvolvimento de sistemas de recomendação. Assim, buscando pensar soluções que possibilitem ao usuário a reivindicação de uma narrativa própria e maior participação em como será avaliado seu perfil, apresentamos a proposta de auto perfil para o cadastro no portal eletrônico do governo, considerando sua integração com o CBC. Através do auto perfil, o usuário pode expressar sua identidade pessoal, tendo maior controle sobre quais informações contidas no CBC serão utilizadas para a formação de seu perfil, além de ser um canal aberto para expressão de outras informações que o usuário considere revelar, garantindo o direito de como ser visto.

\section{Perfil de Usuário para Sistemas de Recomendação}

O perfil de usuário possui uma estrutura que visa obter conhecimento sobre as preferências individuais que determinam o comportamento do usuário. Entende-se preferências como um conjunto de informações sobre adaptação do comportamento do sistema diante das escolhas ou necessidades do usuário. Para desenvolver sistemas de recomendação com indivíduos e máquinas cooperando para executar tarefas, é preciso considerar as características do usuário para construção do modelo ou perfil. De modo geral os modelos construídos são de usuários padrões, para desenvolvimento de modelos personalizados, onde são coletados dados sobre o desempenho médio das pessoas sobre várias tarefas e em vários ambientes de acordo com o seu perfil [Barth 2010].

\subsection{Criação do Perfil de Usuário}

A criação de perfil de usuário utiliza dois modelos para colher dados: informações implícitas e informações explícitas [Montaner et al. 2003]. No modelo de informações implícitas, os dados 
são coletados pelo sistema sem a percepção ou autorização do usuário. Esses dados ou informações são coletadas enquanto o usuário navega no sistema e podem ser: tempo de visita a uma página, movimento do mouse, log de navegação, entre outros [Castellano et al. 2007].

No modelo de informações explícitas, os dados são fornecidos intencionalmente, ou seja, o usuário informa o que realmente deseja para a criação do seu perfil e geralmente isso ocorre através do preenchimento de formulário. Nesse modelo, as informações colhidas são consideradas mais confiáveis por alguns autores, tendo em vista que é o próprio usuário quem fornece e atualiza seus dados, porém, esse modelo cria a dependência do esforço do usuário que nem sempre está disponível para fornecer as informações necessárias, o que torna a interação um pouco difícil [Montaner et al. 2003], [Castellano et al. 2007].

De acordo com [Barth 2010] e [Montaner et al. 2003], as principais técnicas utilizadas para auxiliar a criação do perfil, de forma implícita ou explícita, são:

- Perfil inicial vazio: nessa técnica o perfil do usuário é preenchido automaticamente no início da interação do mesmo com o sistema, de acordo com sua navegação.

- Perfil manual: nessa técnica o usuário digita os seus interesses em um formulário de palavras chaves no sistema que ajuda também na criação do auto perfil de forma segura, pois é criado um perfil com base em informações cedidas e não em informações retiradas sem autorização. O problema é que essa técnica depende totalmente do usuário para fornecer as informações sobre seu interesse que nem sempre ele mesmo sabe descrever.

- Estereótipos: técnica de criação de perfil baseada na criação de um modelo inicial. Os dados utilizados são os dados pessoais, dados geográficos entre outros.

\subsection{Proposta de criação de Auto Perfil no Portal do Governo Eletrônico para recomendar serviços aos cidadãos}

Para a criação do auto perfil, sugerimos a utilização do modelo de informações explícito, que se relaciona a recomendações reativas e proativas, com a possibilidade de utilizar a técnica de perfil manual, e quando não for possível utilizar esse modelo sugerimos a adoção de técnicas de recomendação baseada nos serviços mais acessados no portal.

- Explícito (Perfil Manual) - esse perfil fornece privacidade ao cidadão e permite que ele forneça as informações desejadas, ao invés de ter suas informações colhidas sem autorização ou conhecimento, pois cria o perfil através do preenchimento manual de um formulário.

O formulário, que deverá ser fornecido no portal do governo eletrônico, será utilizado para o cadastro do usuário, através da integração com o $\mathrm{CBC}$, as informações já existentes deverão ser carregadas no perfil do usuário de acordo com as suas preferências. Além disso, o formulário deverá relacionar a lista de serviços do governo para que o usuário selecione as informações que tem interesse ou necessidade de acompanhar, e também a lista das bases que o usuário gostaria de fornecer às suas informações, inclusive dados pessoais e geográficos.

Dessa forma teríamos um portal com duas das possibilidades sugeridas no artigo de [Ayachi et al. 2016], adaptada para o portal do governo do Brasil conforme exemplifica a Figura 1. 


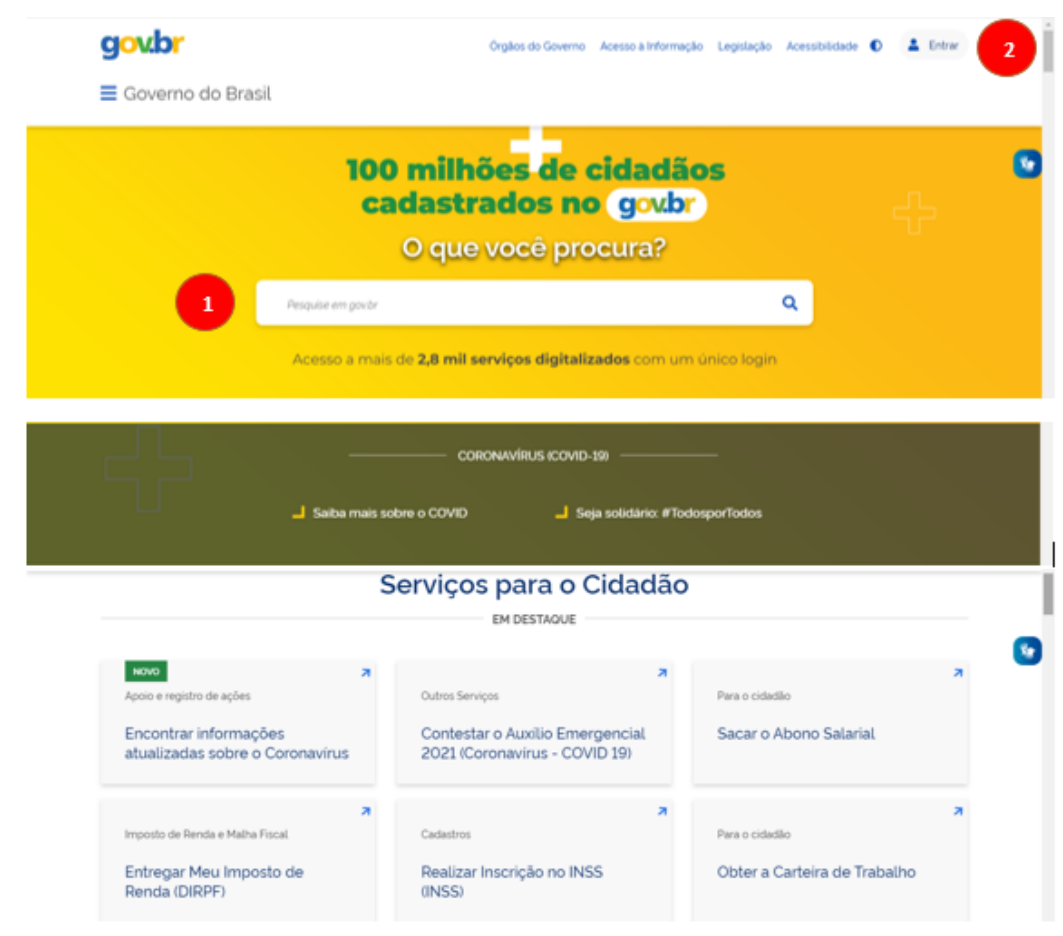

Figura 1. Portal do governo do Brasil

1- O portal apresenta o campo de busca para que o usuário procure por assuntos e serviços de interesse na área não logada a partir de uma lista de serviços mais utilizados;

2- O portal permite a personalização das recomendações para o usuário de acordo com as informações fornecidas no formulário que foi preenchido, utilizando o perfil manual.

Em resumo o modelo de auto perfil sugerido para o Governo Eletrônico seria o explícito com a técnica de perfil manual, porque além das questões de privacidade e proteção dos dados, informadas anteriormente, o usuário terá um login personalizado, com recomendações de serviços de acordo com o seu interesse e necessidade e suas informações só ficarão disponíveis para as bases de dados que realmente importam pra ele. Essa abordagem focada no usuário busca garantir maior autodeterminação informativa ao cidadão.

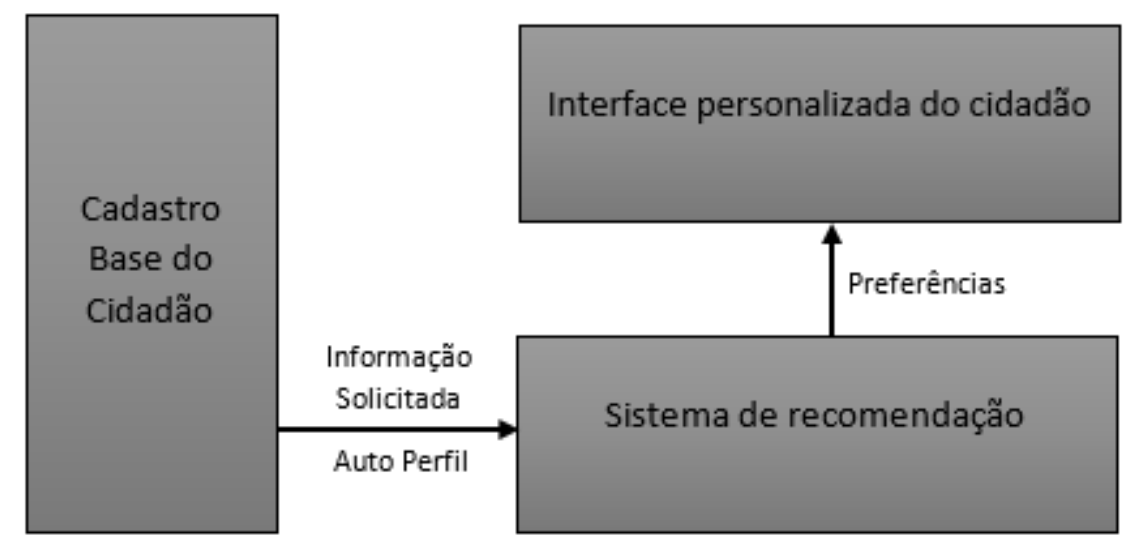

Figura 2. Arquitetura simplificada para a estrutura do e-Gov com perfil personalizado

A Figura 2 apresenta a proposta de arquitetura para sistema de recomendação com perfil personalizado voltado ao portal e-Gov, onde o CBC fornece as informações solicitadas 
de acordo com o auto perfil para o sistema de recomendação, que por sua vez retorna as preferências para a criação da interface personalizada do cidadão.

\section{Modelo Conceitual do Sistema de Recomendação}

Nesta seção apresentaremos o modelo conceitual de um sistema de recomendação de serviços de governo eletrônico (e-Gov) aos cidadãos, descrevendo a função de cada módulo do sistema e como, a partir do auto perfil do usuário, chegaremos à lista de recomendação dos serviços.

Com o intuito de melhorar a experiência do usuário e buscar um resultado mais assertivo do sistema de recomendação usamos uma abordagem híbrida no nosso modelo, combinando diferentes técnicas de recomendação como Collaborative Filtering, Content-based e Contextual Recommendation, de acordo com o que foi sugerido por [Burke 2007], utilizando informações dos bancos de dados para recomendar serviços personalizados e acessíveis aos usuários de acordo com suas necessida

Como pode ser visto na Figura 3, o usuário terá uma interface com o sistema possibilitando a configuração do seu perfil, tema tratado na seção anterior, bem como possibilita a procura por serviços disponíveis na base de dados e onde também receberá as recomendações geradas pelo sistema.

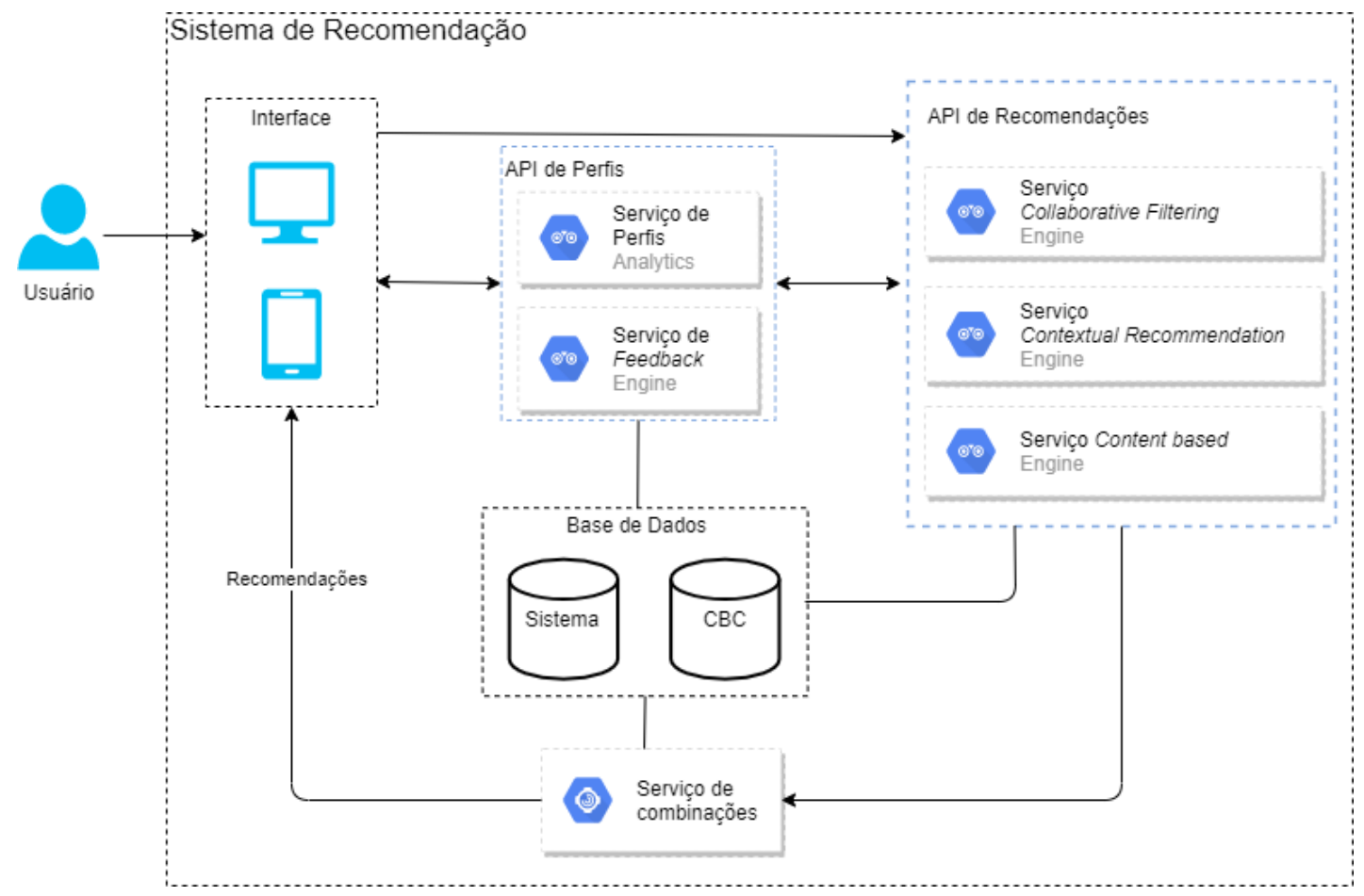

Figura 3. Modelo conceitual para sistemas de recomendação de serviços e-Gov

\subsection{Collaborative Filtering Service}

De acordo com [Schafer et al. 2007] as técnicas de Collaborative Filtering são processos de filtrar ou avaliar itens usando as opiniões de outras pessoas, sendo umas das técnicas mais conhecidas dos sistemas de recomendação. 
O serviço usará o feedback, tema que será explicado no item 5.5, dos usuários com perfil semelhante ao do usuário logado para montar uma matriz esparsa de usuários e suas avaliações dos serviços, conforme apresentado na Tabela 1. A partir da matriz ele montará uma lista de recomendações com base nas avaliações de serviços desses perfis. A ideia é que se um usuário com o perfil semelhante utilizou um determinado serviço pode ser que seja interessante para ele também.

A lista de recomendação será apresentada na ordem decrescente com base nos valores do cálculo da média, ignorando os valores nulos, das avaliações dos usuários sobre os serviços, conforme modelo sugerido por [El Alami et al. 2015].

Tabela 1 - Exemplo da matriz de classificação

\begin{tabular}{|c|c|c|c|}
\hline & Serviço i & Serviço j & Serviço $\mathrm{n}$ \\
\hline User a & 2 & 4 & 1 \\
\hline$\ldots$ & & & \\
\hline User j & 1 & 2 & t) \\
\hline$\ldots$ & & & \\
\hline User $p$ & 2 & 1 & 0 \\
\hline
\end{tabular}

\subsection{Content-based Service}

[Pazzani 2007] define a técnica de Content-based como métodos que recomendam um item para um usuário com base em uma descrição do item e um perfil de interesses do usuário, analisando palavras chaves nas descrições dos itens para identificar itens semelhantes aos já consumidos por ele. A ideia é que se o usuário utilizou um determinado serviço o sistema recomendará serviços similares, por exemplo se o usuário utilizou o serviço de emissão de identidade, pode ser que ele precise do serviço de emissão de carteira de trabalho.

O serviço usará o Cadastro Base do Cidadão $(\mathrm{CBC})$ para verificar quais serviços foram utilizados pelo usuário e fará as recomendações com base no histórico de uso dos serviços que constarem na base e também pelos itens já avaliados no sistema. A ordem de classificação será feita apresentando os serviços semelhantes aos mais recentes encontrados na base.

\subsection{Contextual Recommendation Service}

Segundo [Adomavicius and Tuzhilin 2015] a técnica de recomendação baseadas em contexto geram recomendações que correspondem a um contexto atual, dando pouco peso ao histórico e perfil do usuário. Ele cita que as pessoas possuem necessidades momentâneas e os sistemas de recomendações precisam ser capazes de lidar com isso de maneira inteligente, já que os usuários interagem com o sistema dentro de um contexto particular e que as preferências por itens dentro de um contexto podem ser diferentes daquelas em outro contexto. O objetivo do Contextual Recommendation Service é analisar o relato textual das necessidades momentâneas do usuário e ser capaz de gerar recomendações dentro desses contextos.

Esta proposta é baseada no modelo descrito por [Pardos and Jiang 2020] que descreve como utilizou técnicas de processamento de linguagem natural (PLN) para gerar um sistema de recomendação de cursos universitários, gerando um vetor de palavras chaves a partir da ementa dos cursos. [Rodrigues 2017] explica as tarefas de processamento textual, como normalização, stopwords, remoção de numerais, correção ortográfica, stemming e 
lematização, utilizadas no PLN para gerar um vetor de palavras chaves a partir de um texto. $\mathrm{O}$ serviço usará esse vetor de palavras para comparar com as palavras chaves da descrição dos serviços, buscando semelhança entre elas e então montar a lista de recomendação para o usuário, prática já utilizada no Content-based Service.

É importante observar que esses relatos textuais não farão parte do perfil do usuário, já que a intenção desse serviço é atender as necessidades momentâneas dos usuários dentro do contexto analisado.

\subsection{Reativo e Proativo}

Conforme definido por [Ayachi et al. 2016)] os sistemas de recomendações que propõem funcionalidades reativas e proativas combinam várias técnicas de recomendação que usam diferentes fontes de dados, como perfil do cidadão, bancos de dados de mídia social, bancos de dados de feedback do cidadão e bancos de dados de serviço, para recomendações de serviços de governo eletrônico. O serviço reativo trabalha sobre as necessidades explícitas do cidadão, ou seja, o usuário precisa interagir com o sistema, seja por feedback ou formulários, para que então ele receba as recomendações. Já o mecanismo proativo fornece serviços sem qualquer solicitação. $\mathrm{Na}$ verdade, essa estrutura apresenta um novo aspecto nas técnicas de personalização do governo eletrônico que explora as informações capturadas do CBC. Ele notificará os cidadãos sobre os serviços que podem corresponder aos seus perfis sempre que o sistema encontrar alguma alteração na base de serviços.

O serviço proativo fará o monitoramento apenas dos serviços autorizados pelo usuário, já que a sua privacidade será respeitada.

\subsection{Feedback}

Os feedbacks serão coletados por meios explícitos e implícitos. Feedbacks explícitos são aquelas em que um usuário é solicitado a fornecer uma opinião sobre um item. As opções de respostas serão "Útil" ou "Não útil" e os dados implícitos serão gerados pelo sistema caso seja detectado a utilização de algum serviço no CBC e não for encontrado um feedback explícito para o mesmo serviço. Para as respostas "Não útil" será atribuído o valor 0, para "Útil" o valor 2 e 1 para os implícitos, para as outras situações o valor será nulo. Ao observar a Tabela 1 podemos visualizar como ficará a classificação dos feedbacks.

\section{Conclusão}

A personalização de serviços através do auto perfil, obtida por meio dos sistemas de recomendação, permite que o portal do governo eletrônico se torne mais atrativo para os cidadãos, oferecendo uma interface personalizada de acordo com as preferências do cidadão, com recomendações de acordo com sua necessidade. Outro grande ganho é a proteção dos dados, privacidade e o direito de poder criar o seu próprio perfil fornecendo as informações que deseja ao invés de ter as informações retiradas sem autorização.

O auto perfil pode apoiar a construção de um ambiente mais saudável para o desenvolvimento das identidades pessoais, pois o usuário pode expressar-se através de um maior controle sobre quais informações do Cadastro Base do Cidadão (CBC) serão utilizadas para a formação de seu perfil, visando garantir ao usuário o direito de autodeterminação informacional. Por fim, apresentamos o modelo de um sistema de recomendação híbrido, com 
a combinação de diferentes técnicas de recomendação e com comportamentos reativos e proativos de acordo com o auto perfil e interesses do cidadão.

Para trabalhos futuros podemos sugerir pesquisas relacionadas à aplicação do auto perfil para recomendações personalizadas, utilizando informações fornecidas pelos cidadãos com restrições de privacidade com base em diferentes fontes de dados, tendo em vista que as informações do e-Gov estão espalhados em diversos órgãos públicos. Visto que esse modelo pode gerar erros que forçam o recomendador, baseado em restrições, a fornecer recomendações ruins ou vazias, identificar uma abordagem para solucionar este possível problema será um desafio.

\section{Referências}

Adomavicius, G., \& Tuzhilin, A. (2011). Context-aware recommender systems. In Recommender systems handbook (pp. 217-253). Springer, Boston, MA.

Ayachi, R., Boukhris, I., Mellouli, S., Amor, N. B., \& Elouedi, Z. (2016). Proactive and reactive e-government services recommendation. Universal Access in the Information Society, 15(4), 681-697.

Barth, F. J. (2010). Modelando o perfil do usuário para a construção de sistemas de recomendação: um estudo teórico e estado da arte. Revista de Sistemas de Informação da FSMA, 6, 59-71.

Brasil/a. Presidência da República. Decreto no 10.046, de 9 de outubro de 2019. Dispõe sobre a governança no compartilhamento de dados no âmbito da administração pública federal e institui o Cadastro Base do Cidadão e o Comitê Central de Governança de Dados. Disponível em: <http://www.planalto.gov.br/ccivil_03/_Ato2019-2022/2019/Decreto/D10046.htm>. Acesso em: 26 de janeiro de 2021.

Burke, R. (2007). Hybrid web recommender systems. The adaptive web, 377-408.

Castellano, G., Fanelli, A. M., Mencar, C., \& Torsello, M. A. (2007, November). Similarity-based fuzzy clustering for user profiling. In 2007 IEEE/WIC/ACM International Conferences on Web Intelligence and Intelligent Agent Technology-Workshops (pp. 75-78). IEEE.

Cazella, S. C., Nunes, M. A. S. N., \& Reategui, E. (2010). A Ciência da Opinião: Estado da arte em Sistemas de Recomendação. André Ponce de Leon F. de Carvalho; Tomasz Kowaltowski..(Org.). Jornada de Atualização de Informática-JAI, 161-216.

El Alami, Y. E. M., El Habib Nfaoui, \& El Beqqali, O. (2015). Improving Neighborhood-Based Collaborative Filtering by a Heuristic Approach and an Adjusted Similarity Measure. In BDCA (pp. 16-22).

Ferreira, L. M. T. (2020). Parecer sobre a legalidade dos Decretos n ${ }^{\circ}$ 10.046/2019 e $10.047 / 2019$ em face das normas que disciplinam os direitos fundamentais à proteção de dados e à privacidade no ordenamento jurídico brasileiro. Revista do Ministério Público do Estado do Rio de Janeiro, 65, 257.

Gil, A. C. (2002). Como elaborar projetos de pesquisa (Vol. 4, p. 175). São Paulo: Atlas. 
Governo de Quebec, Portal Eletrônico. Disponível em: <https://www.quebec.ca/>. Acesso em: 26 de Janeiro de 2021.

Governo do Brasil, Portal Eletrônico. Disponível em: <https://www.gov.br/pt-br>. Acesso em: 26 de Janeiro de 2021.

Governo do Brasil/a, Portal Eletrônico. Disponível em: $<$ https://www.gov.br/governodigital/pt-br/governanca-de-dados/cadastro-base-do-cidadao-cbc >. Acesso em: 26 de Janeiro de 2021.

Governo do Brasil/b, Portal Eletrônico. Disponível em: $<$ https://www.gov.br/governodigital/pt-br/EGD2020>. Acesso em: 26 de janeiro de 2021.

Junior, V. F., Ceci, F., Woszezenki, C. R., \& Leopoldo, A. (2017). Design Science Research Methodology Enquanto Estratégia Metodológica para a Pesquisa Tecnológica. Revistas Espacios 38 (6), 25.

Martins, P., \& Hosni, D. (2019). O Livre Desenvolvimento da Identidade Pessoal em Meio Digital: Para além da proteção da privacidade? (The Free Development of Personal Identity in the Digital Environment: Beyond the Privacy Protection?). MARTINS, Pedro, 46-5.

Montaner, M., López, B., \& De La Rosa, J. L. (2003). A taxonomy of recommender agents on the internet. Artificial intelligence review, 19(4), 285-330.

Pardos, Z. A., \& Jiang, W. (2020, March). Designing for serendipity in a university course recommendation system. In Proceedings of the tenth international conference on learning analytics \& knowledge (pp. 350-359).

Pazzani, M. (2007). Content based Recommendation Systems [in:] The Adaptive Web: Methods and Strategies of Web Personalization, eds. P. Brusilovsky, A. Kobsa, W. Nejdl.

Rodrigues, J. (2017). O que é o Processamento de Linguagem Natural? Disponível em: $<$ https://medium.com/botsbrasil/o-que-é-o-processamento-de-linguagem-natural-49ece9371cf f>. Acesso em: 15 jan. 2020.

Schafer, J. B., Frankowski, D., Herlocker, J., \& Sen, S. (2007). Collaborative filtering recommender systems. In The adaptive web (pp. 291-324). Springer, Berlin, Heidelberg. 